\title{
The muscle-bone unit in adolescent swimmers
}

Alejandro Gomez-Bruton ${ }^{1,2,3,4}$; Alejandro Gonzalez-Aguero ${ }^{1,2,3,5,8}$; Angel Matute-Llorente ${ }^{1,2,3,5}$; Gabriel Lozano-Berges $^{1,3,5}$, Alba Gomez-Cabello ${ }^{1,2,3,6,8}$, Luis A. Moreno, ${ }^{1,2,7,8}$; Jose A. Casajus $^{1,2,3,5,8}$; Germán Vicente-Rodríguez ${ }^{1,2,3,5,8}$

${ }^{1}$ GENUD (Growth, Exercise, NUtrition and Development) Research Group, Zaragoza, Spain

${ }^{2}$ Centro de Investigación Biomédica en Red de Fisiopatología de la Obesidad y Nutrición (CIBEROBN), Madrid, Spain

${ }^{3}$ EXERNET red de investigación en ejercicio físico y salud para poblaciones especiales

${ }^{4}$ Universidad Isabel I, Burgos, Spain

${ }^{5}$ Faculty of Health and Sport Science (FCSD), Department of Physiatry and Nursing. Universidad de Zaragoza, Ronda Misericordia 5, 22001-Huesca, Spain.

${ }^{6}$ Centro Universitario de la Defensa, Zaragoza, Spain

${ }^{7}$ Faculty of Health Science (FCS). Universidad de Zaragoza. Zaragoza, Spain

${ }^{8}$ Instituto Agroalimentario de Aragón (IA2), Zaragoza, Spain

\section{Corresponding author:}

German Vicente-Rodriguez, PhD.

GENUD (Growth, Exercise, NUtrition and Development) Research Group

Faculty of Health and Sport Sciences

University of Zaragoza

Grupo GENUD Edificio SAI $2^{\mathrm{a}}$ planta, Zaragoza

Phone: +34 876553755 or 976761000 (Ext. 843754)

Fax: +34974238422 / +34974239384

Email: gervicen@unizar.es 


\section{Abstract}

Purpose: To examine the functional and structural muscle-bone unit in adolescent swimmers.

Methods: Sixty-five swimmers (34 girls/31 boys) and 119 controls (51 girls/68 boys) participated in the study. Muscle cross-sectional area (MCSA), bone mineral content (BMC) and polar strength-strain index (SSIPOL) were measured in the non-dominant radius by peripheral quantitative computed tomography (pQCT). Subtotal BMC and lean mass were evaluated with dual-energy X-ray absorptiometry (DXA). Handgrip and isometric knee extension (IKE) tests were performed to determine muscle force. The effect of MCSA, lean and force on SSIPOL and BMC were tested, and the functional and structural musclebone ratios of swimmers and controls were compared.

Results: Both muscle size (MCSA and lean) and muscle force (hangrip and IKE) influenced BMC and SSIPOL in swimmers and controls similarly. Swimmers presented normal MCSA and lean values for their height, but when compared to controls, swimmers presented a higher amount of lean and MCSA for the same BMC or SSIPOL (structural muscle-bone unit). For the functional muscle-bone unit, different results were found for the lower and upper limbs, as no differences were found for the upper limbs, while for the lower limbs, swimmers presented higher muscle force for the same amount of BMC.

Conclusions: The contradictory results regarding BMC in swimmers found in previous studies could partly be explained with the findings of the present study that reinforce the idea that swimming is not an effective sport to practice regarding bone mass, and that the muscle bone-unit is different in swimmers than in controls.

Keywords: Musculoskeletal; Body composition; Bone Health; Children; Exercise; Swimming 


\section{Introduction}

The positive effects of physical activity and exercise on bone mineral density (BMD) and bone strength have been recently graded with an A and B level of evidence respectively by the National Osteoporosis Foundation[1]. These positive effects are thought to be due to the mechanical forces that are generated either through impact with the ground (i.e. gravitational or ground-reaction forces) or through skeletal muscle contractions (i.e. muscle forces) performed while engaging in different physical activities. It is nowadays clear that different sports have distinct effects on bone mass, with those without groundreaction forces like swimming[2] or cycling[3] showing the weakest effect on BMD or bone mineral content (BMC).

In order to try to explain the positive effect of exercise on bone, Frost designed the Mechanostat theory which predicts that an increase in muscle mass or force during growth would create a moderate overload, which would lead to an increase in bone mass or strength[4]. Consequently, many studies adjust BMD or BMC by lean or muscle mass when comparing athletes to controls. These adjustments are done with the premise that lean affects bone equally in both groups (athletes and controls), and are very important as they can change results substantially. For example, in a previous study in which we compared BMD and BMC in swimmers and controls[5], we found few differences between groups (some of them favouring the swimmers), whereas when data were adjusted, swimmers presented lower BMD values for most of the measured variables. In this line, a recent meta-analysis evaluating the effects of swimming on BMD suggested that swimming might increase lean mass without increasing BMD[6], which could entail that the muscle-bone association is different in swimmers than in other athletes and controls.

In order to study the muscle-bone association, Schoenau et al.[7] proposed and algorithm that took height, $\mathrm{BMC}$ and muscle into account with the aim of describing the muscle-bone unit in different populations. Two different muscle-bone units can be described. The first one is the structural muscle bone unit in which a ratio of bone and muscle mass is estimated. This structural muscle-bone unit has been criticized as muscle force is not necessarily proportional to muscle size[8]. Consequently, some researchers have advocated for the functional muscle-bone unit which is a ratio of bone and muscle force[8]. Although in other athletic disciplines $[9,10]$ and in non-athletes $[8,11]$ this muscle-bone association has been deeply studied, there is a lack of literature exploring this association in adolescent swimmers. It is extremely 
important to evaluate this muscle-bone association, as if muscle is affecting bone differently in swimmers than in other athletes or sedentary controls, adjusting by muscle or lean mass when comparing these groups could be incorrect.

Therefore, the aims of the present study were to evaluate if swimmers present a similar functional and structural muscle-bone unit when compared to controls.

\section{Material and methods}

Study design, protocol and consent forms were performed in accordance with the Helsinki Declaration of 1964 (revised in Fortaleza, 2013) and were reviewed and approved by the Research Ethics Committee of the Government of Aragon (ref. CP08/2012, CEICA, Spain). Sample size calculations for the present project have been explained elsewhere[12], and the sample size is similar to that presented in previous articles evaluating the muscle-bone unit in children and adolescents[13, 14].

\section{Participants}

All participants had to be between the ages of 11 and 18 years, Caucasian, non-smokers, with no chronic disease or musculoskeletal disorders (fibromyalgia, gout, osteoarthritis, rheumatoid arthritis, tendinitis), bone fractures or medication. Swimmers had to have a history of swimming and competing in regional tournaments for more than 3 years and current training for a minimum of 6 hours per week. The normoactive controls (CG) could not be performing any aquatic activity on a regular basis or more than 3 hours of weight-bearing physical activity per week. Swimmers were recruited from 4 swimming clubs while controls were recruited from 4 schools and high-schools of Zaragoza (Spain).

\section{Pubertal stage and anthropometric measurements}

Pubertal maturation was determined by self-assessment of secondary sexual characteristics according to the criteria devised by Tanner[15]. This method has been reported to be both valid and reliable in assessing sexual maturity among adolescent athletes[16].

Height was measured with a stadiometer to the nearest $0.1 \mathrm{~cm}$ (SECA 225, SECA, Hamburg, Germany), and weight with a scale to the nearest $0.1 \mathrm{~kg}$ (SECA 861, SECA, Hamburg, Germany). Both 
anthropometric measures were taken without shoes and minimal clothing following the procedures by International Society for the Advancement in Kinanthropometry (ISAK)[17].

\section{Muscle force}

Maximum isometric forearm force was determined with a digital handgrip dynamometer (Takei TKK 5401, Takei scientific instruments, Tokyo, Japan). Participants were instructed to exert their maximal grip force with the upper limb in extension in two attemps with each hand, with one minute pauses between them. The dynamometer was adapted for each participant according to their hand span as proposed by Ruiz et al.[18] The best attempt was selected for further analysis.

Maximum isometric peak torque through knee extension (IKE) was measured using a strain gauge (MuscleLab, Force Sensor, Norway). The participant was sitting with an anchorage placed on the distal third of the tibia. This anchorage was connected to the strain gauge, registering force data during the 6 seconds that the participant performed the maximum knee extension. Two attempts were allowed for each leg, recording the best performance.

\section{Bone assessments}

Whole body dual-energy X-ray absorptiometry (DXA) scans were performed with the pediatric version of the QDR-Explorer software (Hologic Corp. Software version 12.4. Bedford. Masssachusettes, USA). Outcomes of interest were BMC and lean mass of the subtotal body (whole body - head) and upper- and lower-limbs (obtained from the whole-body scan). All the DXA analyses were performed by the same operator (AML). The coefficients of variation of the DXA in our laboratory for a whole body scan were $2.3 \%$ for BMC and $1.9 \%$ for lean mass. For DXA, the final sample consisted of 65 swimmers (34 girls / 31 boys) and 119 controls (51 girls / 68 boys).

The radius was also measured with a stratec XCT-2000 L peripheral quantitative computed tomography (pQCT) scanner (Stratec Medizintechnik, Pforzheim, Germany). Scanning procedure and coefficients of variation in our laboratory have been described in detail elsewhere[19]. For the present study, data of the $66 \%$ of the total radius length is presented. The same researcher (AML) performed all the scans. Firstly, a scout view was performed to manually locate a reference line on the distal end of the radius. The reference line was placed at the endplate of the radius as suggested by the Stratec XCT-2000 manual. 
Radius total BMC, polar bone strength strain index (SSIPOL; an estimate of the resistance to bending and torsion of the bone)[20], and muscle cross-sectional area (MCSA), were evaluated. As some of the participants presented moved scans, the sample with good quality pQCT scans consisted of 55 swimmers (29 girls / 26 boys) and 88 controls (39 girls /49 boys).

\section{Statistical analyses}

Independent t-tests were performed to compare anthropometric data between swimmers and controls.

Linear regressions were performed to evaluate the association between height and muscle mass and to determine if this association was similar in controls and swimmers. Further linear regressions were performed to test the influence of muscle size (MCSA or lean) or muscle force (handgrip or IKE) on bone (BMC or SSIPOL), and determine if this influence was similar in controls and swimmers.

Lean mass, BMC, and MCSA mean values from the control group (dividing the controls into height groups spanning $10 \mathrm{~cm}$ each) were used as a reference, and five different age- and pubertal-adjusted ratios (a. BMC to MCSA; b. BMC to Handgrip; c. BMC to Lean; d. SSIPOL to MCSA; e. SSIPOL to Handgrip) were calculated for this group. Z-scores were after calculated for the swimmer group with the previously described control values. To evaluate whether a parameter was significantly different from the result in controls, the difference of the mean $\mathrm{Z}$ score to zero was assessed with one-sample t-tests. A significant difference was assumed when the $95 \%$ CI of the mean Z-score did not include zero.

For all analyses, statistical significance was set as $\mathrm{p}<.05$. All analyses were performed with SPSS version 21.0 .

\section{Results \\ Descriptive characteristics}

Anthropometric characteristics are summarized in Table 1. No age differences were found between groups ( $p>.05$; Table 1). Male swimmers were taller and heavier than controls $(\mathrm{p}<.05$; Table 1).

Female swimmers presented lower leg BMC values when compared to controls ( $\mathrm{p}<.05$; Table 1). No other difference was found between groups for any of the measured bone variables independently of the used device (DXA or pQCT). Male swimmers presented higher values of subtotal and arms lean than controls (both $\mathrm{p}<.05$; Table 1). 
Influence of height, lean, force and group (swimmer or control) on bone mineral content and bone strength

Height was positively associated with MCSA and lean mass in both girls and boys ( $<<.05$; data not presented). No significant group by height interactions were found, suggesting that height influenced lean and MCSA similarly in both groups (swimmers and controls).

All muscle (MCSA and lean) and force variables (handgrip and IKE) were positively associated with BMC (measured with DXA and pQCT) and SSIPOL (pQCT) in both girls and boys (all p<.05; Table 2). Regarding the effect of group, both male and female swimmers presented lower values than controls for subtotal, arms and legs BMC measured with DXA when adjusted by lean (Table 2). Nonetheless, when adjusting arms BMC by muscle force these differences disappeared ( $p>.05$; Table 2) for both genders. When adjusting legs BMC by IKE the differences between groups also disappeared for males. No differences were found between groups for BMC or SSIPOL measured with pQCT (all p>.05; Table 2).

\section{Schoenau algorithm}

Data from Tables 3 and 4 were used to calculate z-scores and apply the Schoenau algorithm[7].

For step 1 of the Schoenau muscle-bone unit[7] (is muscle adequate for height?), sex- and height-specific Z-scores were computed with data from Table 3. The mean height-dependent Z-score of subtotal lean and MCSA were not significantly different from zero in the swimmers (both $\mathrm{p}>.05$; Table 5), suggesting that muscle mass (MCSA or Lean mass) was adequate for height in swimmers.

Regarding step 2 of the Schoenau muscle-bone unit[7] (is BMC adequate for muscle mass or force?), all the structural muscle-bone unit ratios were significantly lower in swimmers than in controls (both age and Tanner dependent $\mathrm{p}<.05$; Table 5), suggesting that BMC was not adequate for muscle mass in swimmers. Regarding the functional muscle-bone unit, the legs BMC/force ratio was lower in swimmers than in controls (both age and Tanner dependent $\mathrm{p}<.05$; Table 5), which entails that legs BMC was not adequate for legs muscle force.

\section{Discussion}

The main finding of the present study is that swimmers present lower structural muscle-bone unit ratios, suggesting that they have a lower amount of bone for the same amount of lean mass or higher amount of 
lean mass for the same amount of bone. For the functional muscle-bone unit, different results were found controls, while for the lower limbs, swimmers presented lower BMC for the same amount of muscle force or higher muscle force for the same BMC.

The results found with the linear regressions suggest that both muscle (lean or MCSA) and force (handgrip or IKE) are determinant to BMC and SSIPOL in swimmers. These findings are in line with previous studies that evaluated this association in healthy children and adults[21] and children with different conditions[7]; all finding a strong association between MCSA and BMC. No group by muscle interactions were found, suggesting that the mechanisms regulating the muscle-bone interaction in swimmers and controls are similar. These results were expected, as swimmers are not a population with a specific disease or condition that could regulate their bone-muscle mechanisms in any way as found in other populations[7].

The negative Z-scores found for the structural muscle bone-unit ratios in swimmers, both when taking into account age or Tanner stage could be interpreted in two different ways: 1) swimmers present a higher amount of muscle for each unit of BMC/SSIPOL, or 2) swimmers present a lower amount of BMC and a weaker bone per each unit of lean/MCSA. When focusing on boys, it seems clear that although there are no significant differences between BMC, SSIPOL or MCSA between groups (Table 1), swimmers present higher mean values for MCSA (not significant) and lean (significant). Therefore, swimmers present more MCSA and lean for the same amount of BMC or SSIPOL. This would imply that swimming is effective for improving lean mass but not for stimulating bone mass, at least in the regions in which measurements were performed. This is in line with previous studies that compared swimmers with sedentary controls finding higher lean mass values in the swimmers without higher values in BMC[22] or BMD[23]. This lack of bone improvements in swimmers could be due to two different and not necessarily independent factors; the lack of impact, and the low mechanical strains produced while swimming.

Focusing on impact it has been widely demonstrated that sports that entail high impacts improve bone mass[24-26], and those reviews that included a variety of sports always found that athletes involved in high impact sports presented higher bone values when compared to non-impact sports[27, 28]. Nonetheless, not all impacts have the same effect, as low impacts repeated many times might not be as useful, as demonstrated in a review performed by Tenforde and Fredericson, who showed that athletes 
engaged in high-impact and multidirectional loading sports consistently had greater BMD and geometric bone mass, while the only impacts received by swimmers are those performed while kicking against the wall which will probably entail very low loads.

Regarding the mechanical strains produced while swimming, Meaking et al.[29] described in a review article the mechanisms of adaptation to mechanical loading in bone. Furthermore they described some main points that when specifically analysed and extrapolated to the characteristics of swimming are just the opposite of what the sport demands: 1) "Loading-related bone formation correlates with peak strain magnitude": swimming, like most endurance sports, is characterized by low magnitudes and therefore peak strains will be relatively low; 2) "Increasing strain rate during loading stimulates bone formation": the rate of change of strain magnitude or in other words the acceleration or deceleration which is very low in swimming is also a key factor to bone stimulation; 3) "Number of loading cycles required to maximally stimulate bone formation is small"; relatively few cycles of loading are required to maximize the amount of new bone formed, while in swimming there are thousands of cycles in each training session; 4) "Inserting rest between loading cycles increases bone formation". An animal study by Robling et al.[30] showed that dividing 360 loading cycles into 4 bouts of 90 cycles or 6 bouts of 60 cycles per day enhanced the osteogenic response to loading. Nevertheless, when competing in swimming unlike other sports (i.e. soccer, basketball) there are no rest periods between loading cycles. Therefore, these 4 described points in addition to the lack of eccentric movements in swimming, which have shown to be more effective than concentric movements in improving bone mass[31], could mostly explain why the structural muscle-bone ratios were lower in swimmers than in CG.

When focusing on the functional muscle-bone unit, results where different, as no differences for the $\mathrm{z}-$ scores were found for the $\mathrm{BMC} /$ handgrip and SSIPOL/handgrip ratios, suggesting that swimmers and controls have the same muscle force for the same amount of bone. This does not necessarily imply that swimming is not improving muscle force, as we found that when measuring force with a specific test that simulated swimming stroke, swimmers performed higher amounts of force than controls (unpublished personal findings). Nevertheless, the handgrip test measures the maximal isometric force performed by the forearms and while adolescent swimmers perform many repetitive movements that involve the forearm muscles, they never reach maximum force that, therefore, will probably not be increased as shown in the present study. Surprisingly, the leg functional bone-unit was different between swimmers 
and controls, probably because swimmers presented higher (although not significant) leg muscle force values and similar bone values. The different results found for the upper and lower limb functional muscle-bone unit, could be due to the used non-specific tests to assess muscle force, as although both are unspecific tests, swimmers while performing the turn phase under-water complete a quick and strong push against the wall, which might strengthen the quadriceps (the main muscles involved in the isometric knee extension test). It is possible that if two specific swimming tests had been performed to measure the upper and lower body force, swimmers would have indeed presented higher force values when compared to the controls, and therefore, results for the functional muscle-bone unit would have been similar to the structural muscle bone unit, with swimmers presenting higher muscle force values for the same amount of bone.

Although this study presents several strengths, such as the use of two different devices to measure bone mass, and the estimation of both functional and structural muscle-bone units, it is not extent of limitations, with the main one being the type of muscle force assessment; isometric tests. A type of muscle contraction that is never performed by swimmers while swimming. Additionally, and as explained in previous studies[8] maximum forces that stimulate bone will be reached with eccentric contractions, and not with isometric ones. Therefore, further studies, using other types of strength assessment in swimmers are needed in order to confirm our results.

The present findings could partly explain the inconsistent results regarding bone mass in adolescent swimmers, as although literature is scarce for pQCT studies, there is a vast amount of literature regarding DXA studies with diverse results[2]. This variety of results could partly be explained by the effect of lean mass, as some researchers adjust BMD or BMC by lean mass. The assumption behind this common adjustment is that lean mass influences bone mass in a similar manner in swimmers and CG. However, our data suggest that the ratio BMC to lean is not similar so lean adjustment should be avoided as when comparing swimmers to $\mathrm{CG}$, swimmers will have more lean mass (although the ratio of BMC to lean mass will be lower in the swimmers). Therefore, when adjusting by lean mass, BMC will become lower in the swimmers when compared to the CG due to the effect of the covariate. Thus, it is possible that differences in bone mass might emerge between both groups when those differences in raw data are not present. This happened in the current study, as when comparing swimmers to controls without adjustment no differences were found for bone variables between groups (Table 1), but when groups were compared adjusting by lean, swimmers presented lower BMC values (Table 2). 
According to the muscle-bone algorithm proposed by Schoenau et al.[7], swimmers would be classified as a population with a primary bone defect, as they do present a normal MCSA for their height, but they do not present an adequate BMC for their MCSA/lean. Nevertheless, the mentioned algorithm was proposed for pediatric bone disease, and populations that are studied with it will generally present normal muscle and low bone as found in kidney transplant patients[7], unlike the swimmers of the present study that presented high muscle mass and normal BMC. Consequently, swimmers should not be considered as a population with primary bone defects. Nonetheless, the idea that swimming is not an effective sport to practice regarding bone mass[6] was reinforced by the present study. Swimming coaches should implement weight-bearing trainings in addition to water training in order to try to improve adolescent swimmers bone mass.

\section{Competing interests:}

All authors declare no disclosures

\section{Authors' $^{\prime}$ contributions}

AGB, AML, AGA, AGC and GLB collected all the data. AGB and AGA prepared the database and the statistical analyses and drafted the first manuscript. LM, JAC and GVR conceived the study, participated in its design and obtained funding. GVR and AGB coordinated the study. All authors have read and approved the final version of the manuscript. 


\section{References}

1. Weaver CM, Gordon CM, Janz KF, et al (2016) The National Osteoporosis Foundation's position statement on peak bone mass development and lifestyle factors: a systematic review and implementation recommendations. Osteoporos Int 27:1281-1386

2. Gomez-Bruton A, Gonzalez-Aguero A, Gomez-Cabello A, et al (2013) Is bone tissue really affected by swimming? A systematic review. PLoS One 8:e70119

3. Olmedillas H, Gonzalez-Aguero A, Moreno LA, et al (2012) Cycling and bone health: a systematic review. BMC Med 10:168

4. Burr DB (1997) Muscle strength, bone mass, and age-related bone loss. J Bone Min Res $12: 1547-1551$

5. Gomez-Bruton A, González-Agüero A, Gómez-Cabello A, et al (2015) The effects of swimming training on bone tissue in adolescence. Scand J Med Sci Sport 25:

6. Gomez-Bruton A, Montero-Marín J, González-Agüero A, et al (2016) The Effect of Swimming During Childhood and Adolescence on Bone Mineral Density: A Systematic Review and MetaAnalysis. Sport Med 46: . doi: 10.1007/s40279-015-0427-3

7. Schoenau E, Neu CM, Beck B, et al (2002) Bone mineral content per muscle cross-sectional area as an index of the functional muscle-bone unit. J Bone Min Res 17:1095-1101

8. Anliker E, Toigo M (2012) Functional assessment of the muscle-bone unit in the lower leg. J Musculoskelet Neuronal Interact 12:46-55

9. Ireland A, Maden-Wilkinson T, McPhee J, et al (2012) Upper limb muscle-bone asymmetries and bone adaptation in elite youth tennis players. Med Sci Sport Exerc 45:1749-1758

10. Calbet JA, Moysi JS, Dorado C, Rodriguez LP (1998) Bone mineral content and density in professional tennis players. Calcif Tissue Int 62:491-496

11. Schoenau E (2005) The "functional muscle-bone unit": a two-step diagnostic algorithm in pediatric bone disease. Pediatr Nephrol 20:356-359

12. Gomez-Bruton A, Gonzalez-Aguero A, Gomez-Cabello A, et al (2015) Bone structure of adolescent swimmers; a peripheral quantitative computed tomography (pQCT) study. J Sci Med Sport 19:707-712

13. Veilleux L-N, Pouliot-Laforte A, Lemay M, et al (2015) The functional muscle-bone unit in patients with osteogenesis imperfecta type I. Bone 79:52-57 . doi: 10.1016/j.bone.2015.05.019

14. Soucek O, Matyskova J, Anliker E, et al (2015) The muscle-bone interaction in Turner syndrome. Bone 74:160-165 . doi: 10.1016/j.bone.2015.01.017

15. Tanner JM, Whitehouse RH, Takaishi M (1966) Standards from birth to maturity for height, weight, height velocity, and weight velocity: British children, 1965. II. Arch Dis Child 41:613635

16. Leone M, Comtois AS (2007) Validity and reliability of self-assessment of sexual maturity in elite adolescent athletes. J Sport Med Phys Fit 47:361-365

17. Marfell-Jones M, Olds T, Stewart A, Carter L (2006) International Standards for Anthropometric assessment. International Society for the Advancement of Kinanthropometry (ISAK). Potchefstroom, South Africa

18. Ruiz JR, Espana-Romero V, Ortega FB, et al (2006) Hand span influences optimal grip span in male and female teenagers. J Hand Surg Am 31:1367-1372

19. Gomez-Bruton A, González-Agüero A, Casajus JA, Vicente-Rodríguez G (2014) Swimming training repercussion on metabolic and structural bone development. Benefits of the incorporation 
of whole body vibration or pliometric training. The RENACIMIENTO project. Nutr Hosp 30:399-409

20. Schiessl H, Ferretti JL, Tysarczyk-Niemeyer G, Willnecker J (1996) Noninvasive bone strength index as analysed by peripheral quantitative computed tomography (pQCT). In: Schoenau E (ed) Paediatric osteology: new developments in diagnostics and therapy. Elsevier, Amsterdam, The Netherlands, pp 141-146

21. Schoenau E, Neu CM, Mokov E, et al (2000) Influence of puberty on muscle area and cortical bone area of the forearm in boys and girls. J Clin Endocrinol Metab 85:1095-1098

22. Jurimae J, Cicchella A, Tillmann V, et al (2009) Effect of pubertal development and physical activity on plasma ghrelin concentration in boys. J Endocrinol Invest 32:18-22

23. Ferry B, Lespessailles E, Rochcongar P, et al (2013) Bone health during late adolescence: Effects of an 8-month training program on bone geometry in female athletes. Jt Bone Spine 80:57-63

24. Hagman M, Helge EW, Hornstrup T, et al (2018) Bone mineral density in lifelong trained male football players compared with young and elderly untrained men. J Sport Heal Sci 7:159-168 . doi: 10.1016/J.JSHS.2017.09.009

25. Lozano-Berges G, Matute-Llorente Á, González-Agüero A, et al (2017) Soccer helps build strong bones during growth: a systematic review and meta-analysis. Eur J Pediatr. doi: 10.1007/s00431017-3060-3

26. Burt LA, Greene DA, Ducher G, Naughton GA (2013) Skeletal adaptations associated with prepubertal gymnastics participation as determined by DXA and pQCT: a systematic review and meta-analysis. J Sci Med Sport 16:231-239

27. Tenforde AS, Fredericson M (2011) Influence of sports participation on bone health in the young athlete: a review of the literature. PM\&R 3:861-867

28. Guadalupe-Grau A, Fuentes T, Guerra B, Calbet JA (2009) Exercise and bone mass in adults. Sport Med 39:439-468

29. Meakin LB, Price JS, Lanyon LE (2014) The Contribution of Experimental in vivo Models to Understanding the Mechanisms of Adaptation to Mechanical Loading in Bone. Front Endocrinol $5: 154$

30. Robling AG, Burr DB, Turner CH (2000) Partitioning a daily mechanical stimulus into discrete loading bouts improves the osteogenic response to loading. J Bone Min Res 15:1596-1602

31. Hawkins SA, Schroeder ET, Wiswell RA, et al (1999) Eccentric muscle action increases sitespecific osteogenic response. Med Sci Sport Exerc 31:1287-1292 
Table 1. Anthropometric characteristics, bone and muscle strength variables

\begin{tabular}{|c|c|c|c|c|}
\hline & \multicolumn{2}{|c|}{ Girls $(n=85)$} & \multicolumn{2}{|c|}{ Boys $(n=99)$} \\
\hline & $C G(n=51)$ & SWI $(n=34)$ & $C G(n=68)$ & SWI $(n=31)$ \\
\hline$A g e_{(y)}$ & $14.2 \pm 2.3$ & $13.9 \pm 1.9$ & $14.9 \pm 2.3$ & $15.1 \pm 1.5$ \\
\hline Weight $_{(\mathrm{kg})}$ & $50.9 \pm 10.9$ & $47.8 \pm 8.4$ & $55.9 \pm 12.6^{*}$ & $61.7 \pm 10.1$ \\
\hline Height $_{(\mathrm{cm})}$ & $156.2 \pm 7.8$ & $156.8 \pm 9.1$ & $164.8 \pm 12.4^{*}$ & $171.4 \pm 8.9$ \\
\hline Tanner stage (IIIIIIIIIVIV) & $2 / 9 / 16 / 10 / 14$ & $1 / 8 / 8 / 16 / 1$ & $1 / 10 / 10 / 15 / 32$ & $0 / 2 / 8 / 13 / 8$ \\
\hline \multicolumn{5}{|l|}{$D X A$} \\
\hline Subtot_BMC $(g)$ & $1271.134 \pm 316.906$ & $1188.993 \pm 310.165$ & $1540.004 \pm 482.605$ & $1598.500 \pm 374.931$ \\
\hline $\operatorname{Arms}_{-} \bar{B} M C_{(g)}$ & $101.711 \pm 26.937$ & $99.255 \pm 25.836$ & $121.572 \pm 41.426$ & $135.299 \pm 33.480$ \\
\hline $\operatorname{Legs\_ } B M C_{(g)}$ & $318.505 \pm 75.187 *$ & $283.895 \pm 66.770$ & $402.011 \pm 119.235$ & $404.741 \pm 93.503$ \\
\hline Subtot_Lean $(g)$ & $30591.035 \pm 5779.726$ & $29968.373 \pm 5767.138$ & $38415.18 \pm 9860.463 *$ & $43028.005 \pm 8214.649$ \\
\hline $\operatorname{Legs}_{s_{-}}$Lean $(g)$ & $5680.941 \pm 1116.848$ & $5333.074 \pm 996.291$ & $7274.547 \pm 1863.74$ & $7949.562 \pm 1545.221$ \\
\hline Arms_Lean $_{(g)}$ & $1502.969 \pm 273.985$ & $1569.289 \pm 308.990$ & $2136.03 \pm 634.143^{*}$ & $2517.67 \pm 547.38$ \\
\hline \multicolumn{5}{|l|}{$p Q C T^{\#}$} \\
\hline Radius length $_{(\mathrm{mm})}$ & $232.82 \pm 14.17$ & $234.59 \pm 12.43$ & $252.41 \pm 20.92$ & $259.58 \pm 21.04$ \\
\hline Radius $B M C_{(\mathrm{mg} / \mathrm{mm})}$ & $0.81 \pm 0.14$ & $0.77 \pm 0.14$ & $0.90 \pm 0.22$ & $0.90 \pm 0.15$ \\
\hline Radius SSIPOL $_{(N)}$ & $207.8 \pm 52.53$ & $200.33 \pm 48.7$ & $256.14 \pm 82.57$ & $268.71 \pm 54.09$ \\
\hline $\operatorname{Radius}_{\mathrm{MCSA}}^{\left(\mathrm{mm}^{2}\right)}$ & $2041.19 \pm 295.54$ & $2101.31 \pm 358.14$ & $2748.30 \pm 697.22$ & $2907.58 \pm 630.95$ \\
\hline \multicolumn{5}{|l|}{ Muscle strength } \\
\hline Handgrip $_{(k g)}$ & $22.1 \pm 4.9$ & $22.3 \pm 5.2$ & $29.7 \pm 9.7$ & $32.0 \pm 7.3$ \\
\hline Leg force $(\mathrm{kg})$ & $38.3 \pm 11.5$ & $37.9 \pm 10.6$ & $47.3 \pm 14.5$ & $51.4 \pm 10.4$ \\
\hline
\end{tabular}

Values are mean \pm standard deviation

$* \mathrm{P}<0.05$ Difference between groups within gender.

\# for pQCT variables there were 68 girls (39 controls vs. 29 swimmers) and 75 boys (49 controls vs. 26 boys)

Arms=Mean value of the left and right arm; BMC=Bone mineral content; DXA=Dual energy $\mathrm{X}$-ray; Handgrip=Mean values of the right and left arm;

Leg force $=$ Mean leg force of the left and right legs; Legs=Mean value of the left and right arm; MCSA=Muscle cross-sectional area;

pQCT=Peripheral quantitative computed tomography; SSIPOL=Polar strain strength index; Subtot=Whole body less the head 
Table 2. Results of the different centered linear regressions including both swimmers and controls

\begin{tabular}{|c|c|c|c|c|c|c|c|c|c|}
\hline \multirow{3}{*}{$\begin{array}{l}\text { Independent variables } \\
\text { Girls }\end{array}$} & \multicolumn{5}{|c|}{ Structural muscle bone unit } & \multicolumn{4}{|c|}{ Functional muscle bone unit } \\
\hline & \multicolumn{3}{|c|}{ DXA } & \multicolumn{2}{|c|}{ PQCT } & \multicolumn{2}{|c|}{ DXA } & \multicolumn{2}{|c|}{ PQCT } \\
\hline & Subt. BMC & Arms BMC & Legs BMC & $\mathrm{BMC}_{1}$ & SSIPOL $_{1}$ & Arms BMC & Legs BMC & $\mathrm{BMC}_{2}$ & $\mathrm{SSIPOL}_{2}$ \\
\hline$\beta$ Group & $-0.080^{*}$ & $-0.144^{*}$ & $-0.093^{\star}$ & -0.272 & -0.168 & -0.034 & $-0.208^{*}$ & -0.109 & -0.065 \\
\hline$\beta$ Lean or MCSA & $0.921^{*}$ & $0.921 *$ & $0.876^{*}$ & $0.762 *$ & $0.697 *$ & - & - & - & - \\
\hline$\beta$ Muscle strength & - & - & - & - & - & $0.855^{*}$ & $0.715^{*}$ & $0.788 *$ & $0.785^{*}$ \\
\hline$\beta$ Interaction & -0.011 & -0.066 & -0.001 & -0.100 & -0.050 & -0.021 & 0.048 & 0.019 & -0.021 \\
\hline Model r square & 0.849 & 0.763 & 0.802 & 0.515 & 0.448 & 0.707 & 0.607 & 0.655 & 0.601 \\
\hline Boys & Subt. BMC & Arms BMC & Legs BMC & $\mathrm{BMC}_{1}$ & SSIPOL $_{1}$ & Arms BMC & Legs BMC & $\mathrm{BMC}_{2}$ & $\mathrm{SSIPOL}_{2}$ \\
\hline$\beta$ Group & $-0.145^{*}$ & $-0.100^{*}$ & $-0.141^{*}$ & -0.053 & 0.064 & 0.084 & -0.083 & 0.060 & 0.155 \\
\hline$\beta$ Lean or MCSA & $0.986^{*}$ & $0.977 *$ & $0.971^{*}$ & $0.917^{*}$ & $0.912 *$ & - & - & - & - \\
\hline$\beta$ Muscle strength & - & - & - & - & - & $0.851^{*}$ & $0.799 *$ & $0.842 *$ & $0.808 *$ \\
\hline$\beta$ Interaction & -0.011 & -0.037 & $-0.060-$ & -0.096 & -0.167 & 0.007 & 0.022 & -0.037 & -0.091 \\
\hline Model r square & 0.884 & 0.874 & 0.866 & 0.754 & 0.705 & 0.754 & 0.642 & 0.679 & 0.598 \\
\hline
\end{tabular}

${ }^{*} \mathrm{p}<0.05 ;{ }^{\ddagger} \mathrm{p}<0.07$

$\beta=$ Beta standardized coefficient

Group=A negative symbol indicates that swimmers have lower values than controls

Muscle force=For the arms handgrip and for the legs maximum isometric force for leg extension

Interaction=Interaction between group (SWI or $\mathrm{CG}$ ) and lean mass or muscle force 
Table 3. Height-dependent results for BMC and lean for the subtotal body (whole body less the head), arms and legs of the controls

\begin{tabular}{|c|c|c|c|c|c|c|c|c|c|c|c|c|}
\hline \multirow{2}{*}{$\begin{array}{l}\text { Height } \\
\text { range } \mathrm{cm}\end{array}$} & \multirow[b]{2}{*}{ Girls } & \multirow[b]{2}{*}{ Boys } & \multirow[b]{2}{*}{ Girls } & \multicolumn{3}{|r|}{ DXA } & \multirow{2}{*}{\multicolumn{2}{|c|}{ Boys }} & \multicolumn{4}{|c|}{ pQCT } \\
\hline & & & & Boys & Girls & Boys & & & Girls & Boys & Girls & Boys \\
\hline & & & \multicolumn{2}{|c|}{ Subtotal } & \multicolumn{2}{|c|}{ Arms } & \multicolumn{2}{|c|}{ Legs } & \multicolumn{4}{|c|}{ Radius } \\
\hline & & & \multicolumn{2}{|c|}{$\mathrm{BMC}(\mathrm{kg})$} & \multicolumn{2}{|c|}{$\mathrm{BMC}(\mathrm{kg})$} & \multicolumn{2}{|c|}{$\mathrm{BMC}(\mathrm{kg})$} & \multicolumn{4}{|c|}{$\mathrm{BMC}\left(\mathrm{mg} / \mathrm{mm}^{2}\right)$} \\
\hline$<150$ & 9 & 10 & $0.842 \pm 0.134$ & $0.857 \pm 0.115$ & $0.065 \pm 0.010$ & $0.065 \pm 0.010$ & $0.221 \pm 0.032$ & $0.228 \pm 0.032$ & 8 & 7 & $0.644 \pm 0.087$ & $0.610 \pm 0.095$ \\
\hline $150-160$ & 24 & 15 & $1.210 \pm 0.200$ & $1.212 \pm 0.128$ & $0.098 \pm 0.019$ & $0.089 \pm 0.012$ & $0.299 \pm 0.047 *$ & $0.328 \pm 0.035$ & 21 & 11 & $0.817 \pm 0.119$ & $0.771 \pm 0.099$ \\
\hline $160-170$ & 17 & 18 & $1.563 \pm 0.200$ & $1.551 \pm 0.243$ & $0.124 \pm 0.017$ & $0.127 \pm 0.022$ & $0.390 \pm 0.045$ & $0.401 \pm 0.054$ & 9 & 12 & $0.913 \pm 0.092$ & $0.939 \pm 0.092 *$ \\
\hline $170-180$ & & 16 & & $1.941 \pm 0.339$ & & $0.151 \pm 0.028$ & & $0.501 \pm 0.083$ & & 12 & & $1.014 \pm 0.184$ \\
\hline \multirow[t]{2}{*}{$>180$} & & 9 & & $2.106 \pm 0.313$ & & $0.172 \pm 0.025$ & & $0.542 \pm 0.080$ & & 7 & & $1.124 \pm 0.136$ \\
\hline & & & \multicolumn{2}{|c|}{ Lean $(\mathrm{kg})$} & \multicolumn{2}{|c|}{ Lean $(\mathrm{kg})$} & \multicolumn{2}{|c|}{ Lean $(\mathrm{kg})$} & \multicolumn{4}{|c|}{$\operatorname{MCSA}\left(\mathrm{mm}^{2}\right)$} \\
\hline$<150$ & 9 & 10 & $22.063 \pm 3.139$ & $22.929 \pm 2.483$ & $1.137 \pm 0.142$ & $1.202 \pm 0.142$ & $4.144 \pm 0.629$ & $4.366 \pm 0.593$ & 8 & 7 & $1696.2 \pm 270.9$ & $1750.2 \pm 175.9$ \\
\hline $150-160$ & 24 & 15 & $30.224 \pm 3.793$ & $32.045 \pm 3.117$ & $1.482 \pm 0.185^{*}$ & $1.698 \pm 0.268$ & $5.576 \pm 0.762 *$ & $6.219 \pm 0.653$ & 21 & 11 & $2101.5 \pm 229.2 *$ & $2331.3 \pm 334.3$ \\
\hline $160-170$ & 17 & 18 & $35.252 \pm 3.478^{*}$ & $38.838 \pm 4.303$ & $1.714 \pm 0.222 *$ & $2.176 \pm 0.310$ & $6.534 \pm 0.733^{*}$ & $7.200 \pm 0.751$ & 9 & 12 & $2200.1 \pm 248.7 *$ & $2751.8 \pm 519.2$ \\
\hline $170-180$ & & 16 & & $47.380 \pm 4.892$ & & $2.709 \pm 0.415$ & & $8.970 \pm 0.794$ & & 12 & & $3376.3 \pm 511.2$ \\
\hline$>180$ & & 9 & & $49.453 \pm 5.660$ & & $2.802 \pm 0.285$ & & $9.396 \pm 1.441$ & & 7 & & $3319.1 \pm 220.2$ \\
\hline
\end{tabular}

Values are mean \pm standard deviation

*Significant differences between results in girls and boys of the same height group $(\mathrm{p}<0.05$ in each case)

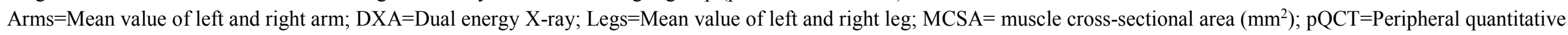
computed tomography; Subtotal=Whole body excluding the head. 
Table 4. Variation with age and Tanner stage of following ratios (only for controls): BMC/lean mass, BMC/force, BMC/MCSA, SSIPOL/MCSA, SSIPOL/Handgrip

\begin{tabular}{|c|c|c|c|c|c|c|c|c|c|c|c|c|}
\hline \multirow{3}{*}{ DXA } & & & \multicolumn{6}{|c|}{ Structural muscle-bone unit } & \multicolumn{4}{|c|}{ Functional muscle-bone unit } \\
\hline & \multicolumn{2}{|c|}{$\mathrm{n}$} & \multicolumn{2}{|c|}{$\begin{array}{c}\text { Subtotal body } \\
\mathrm{BMC}_{(\mathrm{g})} / \text { Lean }_{(\mathrm{kg})} \text { ratio }\end{array}$} & \multicolumn{2}{|c|}{$\begin{array}{c}\text { Arms } \\
\mathrm{BMC}_{(\mathrm{g})} / \mathrm{lean}_{(\mathrm{kg})} \text { ratio }\end{array}$} & \multicolumn{2}{|c|}{$\begin{array}{c}\text { Legs } \\
\mathrm{BMC}_{(\mathrm{g})} / \mathrm{Lean}_{(\mathrm{kg})} \text { ratio }\end{array}$} & \multicolumn{2}{|c|}{$\begin{array}{c}\text { Arms } \\
\mathrm{BMC}_{(\mathrm{g})} / \text { Handgrip }_{(\mathrm{kg})} \text { ratio }\end{array}$} & \multicolumn{2}{|c|}{$\begin{array}{c}\text { Legs } \\
\mathrm{BMC}_{(\mathrm{g})} / \text { Leg force }(\mathrm{kg}) \text { ratio }\end{array}$} \\
\hline & Girls & Boys & Girls & Boys & Girls & Boys & Girls & Boys & Girls & Boys & Girls & Boys \\
\hline \multicolumn{13}{|l|}{$\operatorname{Age}_{(y)}$} \\
\hline$<13$ & 22 & 17 & $39.28 \pm 3.68$ & $37.26 \pm 3.26$ & $60.31 \pm 8.21 *$ & $54.85 \pm 6.37$ & $54.46 \pm 5.69$ & $52.12 \pm 4.67$ & $4.34 \pm 0.74$ & $4.15 \pm 0.54$ & $8.82 \pm 2.16$ & $8.27 \pm 1.56$ \\
\hline $13-15$ & 9 & 15 & $40.86 \pm 3.85$ & $38.89 \pm 2.34$ & $67.36 \pm 8.88^{*}$ & $53.15 \pm 4.69$ & $53.54 \pm 4.32$ & $54.55 \pm 3.70$ & $4.84 \pm 0.97^{*}$ & $3.88 \pm 0.62$ & $9.34 \pm 1.48$ & $8.93 \pm 1.37$ \\
\hline $15-17$ & 11 & 16 & $42.24 \pm 3.08$ & $41.06 \pm 4.40$ & $72.38 \pm 5.50 *$ & $58.72 \pm 6.86$ & $56.58 \pm 5.06$ & $56.08 \pm 5.24$ & $4.86 \pm 0.59$ & $4.36 \pm 0.66$ & $8.31 \pm 1.96$ & $8.22 \pm 0.98$ \\
\hline$>17$ & 9 & 20 & $44.96 \pm 5.18^{\ddagger}$ & $41.18 \pm 3.89$ & $76.47 \pm 9.57^{*}$ & $58.57 \pm 7.23$ & $60.89 \pm 6.24$ & $56.61 \pm 5.93$ & $4.81 \pm 0.31 *$ & $4.02 \pm 0.68$ & $7.57 \pm 1.25$ & $7.96 \pm 1.67$ \\
\hline \multicolumn{13}{|c|}{ Tanner stage } \\
\hline II & 9 & 10 & $38.07 \pm 2.34$ & $39.04 \pm 3.15$ & $56.64 \pm 4.27$ & $55.61 \pm 4.69$ & $55.52 \pm 3.80$ & $54.45 \pm 4.87$ & $3.99 \pm 0.62$ & $4.21 \pm 0.60$ & $8.40 \pm 1.55$ & $8.28 \pm 1.53$ \\
\hline III & 16 & 10 & $41.29 \pm 4.05^{*}$ & $36.99 \pm 3.84$ & $65.57 \pm 7.80 *$ & $53.05 \pm 6.99$ & $53.91 \pm 3.56$ & $52.17 \pm 5.34$ & $4.49 \pm 0.77^{\star}$ & $3.88 \pm 0.66$ & $9.40 \pm 1.87$ & $8.59 \pm 1.51$ \\
\hline IV & 10 & 15 & $42.92 \pm 4.70 *$ & $38.51 \pm 1.99$ & $74.92 \pm 7.39 *$ & $54.43 \pm 6.01$ & $55.45 \pm 6.39$ & $53.94 \pm 3.15$ & $4.82 \pm 0.85^{*}$ & $3.99 \pm 0.54$ & $7.92 \pm 1.73$ & $8.53 \pm 1.62$ \\
\hline $\mathrm{V}$ & 17 & 37 & $43.15 \pm 5.38$ & $41.34 \pm 4.55$ & $72.27 \pm 10.50 *$ & $58.93 \pm 6.73$ & $57.16 \pm 7.81$ & $56.50 \pm 5.79$ & $5.12 \pm 0.40 *$ & $4.19 \pm 0.70$ & $8.04 \pm 1.96$ & $8.18 \pm 1.38$ \\
\hline PQCT & 1 & 1 & $\begin{array}{r}\mathrm{BMC}_{\left(\mathrm{mg} / \mathrm{mm}^{2}\right.} \\
\mathrm{rat}\end{array}$ & $\begin{array}{l}\mathrm{MCSA}_{\left(\mathrm{mm}^{2}\right)} \\
\mathrm{o}^{\Delta}\end{array}$ & $\begin{array}{r}\operatorname{SSIPOL}_{(\mathrm{mm}} \\
\mathrm{ra}\end{array}$ & $\overline{\operatorname{MCSA}_{(\mathrm{kg})}}$ & & & $\begin{array}{r}\mathrm{BMC}_{(\mathrm{mg} / \mathrm{mn}} \\
\mathrm{r}\end{array}$ & Hangrip $_{(\mathrm{kg})}$ & $\begin{array}{r}\mathrm{SSIPOL}_{(\mathrm{mm}} \\
\mathrm{ra}\end{array}$ & Hangrip $_{(\mathrm{kg})}$ \\
\hline \multicolumn{13}{|l|}{ Age } \\
\hline$<13$ & 16 & 11 & $3.75 \pm 0.35^{\#}$ & $3.42 \pm 0.51$ & $0.090 \pm 0.015$ & $0.083 \pm 0.021$ & & & $39.98 \pm 9.13$ & $38.75 \pm 8.99$ & $9.473 \pm 1.982$ & $9.336 \pm 2.862$ \\
\hline $13-15$ & 7 & 11 & $3.88 \pm 0.32^{\#}$ & $3.47 \pm 0.54$ & $0.102 \pm 0.016$ & $0.097 \pm 0.020$ & & & $37.48 \pm 3.22$ & $33.92 \pm 5.21$ & $9.792 \pm 1.473$ & $9.498 \pm 1.774$ \\
\hline $15-17$ & 9 & 12 & $4.04 \pm 0.39 *$ & $3.27 \pm 0.46$ & $0.112 \pm 0.013^{*}$ & $0.093 \pm 0.014$ & & & $37.16 \pm 4.14 *$ & $28.08 \pm 4.13$ & $10.299 \pm 1.093^{*}$ & $7.959 \pm 1.185$ \\
\hline$>17$ & 7 & 15 & $4.38 \pm 0.75^{*}$ & $3.16 \pm 0.35$ & $0.115 \pm 0.029^{\#}$ & $0.097 \pm 0.016$ & & & $37.02 \pm 6.32 *$ & $27.70 \pm 5.37$ & $9.688 \pm 2.250$ & $8.524 \pm 2.168$ \\
\hline \multicolumn{13}{|c|}{ Tanner stage } \\
\hline II & 7 & 5 & $3.71 \pm 0.29$ & $3.73 \pm 0.56$ & $0.092 \pm 0.014$ & $0.094 \pm 0.019$ & & & $39.59 \pm 6.07$ & $44.27 \pm 6.84$ & $9.813 \pm 1.791$ & $11.075 \pm 1.394$ \\
\hline III & 13 & 8 & $3.85 \pm 0.38$ & $3.55 \pm 0.62$ & $0.100 \pm 0.015$ & $0.104 \pm 0.024$ & & & $37.33 \pm 5.27$ & $36.69 \pm 6.80$ & $9.671 \pm 1.679$ & $10.715 \pm 2.349$ \\
\hline IV & 8 & 12 & $4.35 \pm 0.72 *$ & $3.29 \pm 0.30$ & $0.115 \pm 0.029 *$ & $0.087 \pm 0.023$ & & & $38.00 \pm 5.40 *$ & $32.23 \pm 4.73$ & $9.939 \pm 2.015$ & $8.41 \pm 2.194$ \\
\hline $\mathrm{V}$ & 10 & 24 & $3.98 \pm 0.37 *$ & $3.16 \pm 0.40$ & $0.103 \pm 0.018 *$ & $0.092 \pm 0.012$ & & & $36.39 \pm 4.39 *$ & $27.09 \pm 4.21$ & $9.381 \pm 1.746^{*}$ & $7.855 \pm 1.232$ \\
\hline
\end{tabular}

*Significant differences between girls and boys of the same age or Tanner group $(\mathrm{p}<0.05)$.

$\$$ Tendency towards differences between girls and boys of the same age or Tanner group $(\mathrm{p} \leq 0.07)$ 
Table 5. Z-score values for the different analyses

\begin{tabular}{|c|c|c|c|c|c|c|c|}
\hline \multirow{3}{*}{\multicolumn{3}{|c|}{$\begin{array}{l}\text { Subtotal lean - Height dependent } \\
\text { MCSA - Height dependent }\end{array}$}} & \multirow{4}{*}{$\begin{array}{c}\text { Mean dif. } \\
-0.018 \\
0.141\end{array}$} & \multirow{3}{*}{$\begin{array}{c}\text { SE } \\
0.125 \\
0.159\end{array}$} & \multicolumn{2}{|c|}{$\mathrm{CI}$} & \multirow{3}{*}{$\begin{array}{c}\mathrm{p} \\
0.887 \\
0.380\end{array}$} \\
\hline & & & & & -0.267 & 0.231 & \\
\hline & & & & & -0.178 & 0.459 & \\
\hline 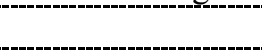 & +1 & \multicolumn{5}{|c|}{ Age dependent $z$-scores } & \\
\hline \multirow{5}{*}{$\begin{array}{l}\text { Structural } \\
\text { muscle-bone unit }\end{array}$} & \multirow{3}{*}{ DXA } & Subtotal BMC/Lean & -0.720 & 0.148 & -1.015 & -0.424 & $<0.001$ \\
\hline & & Arms BMC/Lean & -0.451 & 0.113 & -0.676 & -0.225 & $<0.001$ \\
\hline & & Legs BMC/Lean & -0.744 & 0.151 & -1.045 & -0.444 & $<0.001$ \\
\hline & \multirow{2}{*}{ PQCT } & $\mathrm{BMC} / \mathrm{MCSA}$ & -0.596 & 0.139 & -0.875 & -0.317 & $<0.001$ \\
\hline & & SSIPOL/MCSA & -0.273 & 0.127 & -0.528 & -0.018 & 0.036 \\
\hline \multirow{4}{*}{$\begin{array}{l}\text { Functional } \\
\text { muscle-bone unit }\end{array}$} & \multirow{2}{*}{ DXA } & Arms BMC/Handgrip & 0.095 & 0.122 & -0.149 & 0.339 & 0.440 \\
\hline & & Legs BMC/force & -0.654 & 0.111 & -0.878 & -0.431 & $<0.001$ \\
\hline & \multirow{2}{*}{ PQCT } & BMC/Handgrip & -0.077 & 0.153 & -0.384 & 0.231 & 0.620 \\
\hline & & SSIPOL/Handgrip & 0.076 & 0.156 & -0.2370 & 0.3883 & 0.630 \\
\hline & & \multicolumn{6}{|c|}{ Tanner dependent z-scores } \\
\hline \multirow{5}{*}{$\begin{array}{l}\text { Structural } \\
\text { muscle-bone unit }\end{array}$} & \multirow{3}{*}{ DXA } & Subtotal BMC/Lean & -0.559 & 0.169 & -0.896 & -0.222 & 0.002 \\
\hline & & Arms BMC/Lean & -0.525 & 0.133 & -0.791 & -0.258 & $<0.001$ \\
\hline & & Legs BMC/Lean & -0.646 & $\mathbf{0 . 1 5 7}$ & -0.960 & -0.331 & $<0.001$ \\
\hline & \multirow{2}{*}{ PQCT } & $\mathrm{BMC} / \mathrm{MCSA}$ & -0.622 & $\mathbf{0 . 1 3 8}$ & -0.898 & -0.346 & $<0.001$ \\
\hline & & SSIPOL/MCSA & -0.249 & 0.106 & -0.249 & -0.462 & 0.022 \\
\hline \multirow{4}{*}{$\begin{array}{l}\text { Functional } \\
\text { muscle-bone unit }\end{array}$} & \multirow{2}{*}{ DXA } & Arms BMC/Handgrip & 0.164 & 0.132 & -0.100 & 0.429 & 0.219 \\
\hline & & Legs BMC/force & -0.473 & 0.092 & -0.656 & -0.289 & $<0.001$ \\
\hline & \multirow{2}{*}{ PQCT } & $\mathrm{BMC} /$ Handgrip & -0.186 & 0.140 & -0.467 & 0.096 & 0.191 \\
\hline & & SSIPOL/Handgrip & 0.047 & 0.130 & -0.214 & 0.308 & 0.719 \\
\hline
\end{tabular}

All the z-scores are sex specific.

Mean dif=Mean difference between the groups (Swimmers vs. CG)

$\mathrm{SE}=$ Standard error; $\mathrm{CI}=$ Confidence interval

$\mathrm{p}<0.05$ 